\title{
DYNMAMICAL EFFECTS ON GALAXIES IN CLUSTERS
}

\author{
BEN MOORE, NEAL KATZ AND GEORGE LAKE \\ Department of Astronomy \\ University of Washington \\ Seattle, WA 98195, USA
}

\section{INTRODUCTION}

For nearly 20 years, we've known that clusters at $z \gtrsim 0.3$ have a substantial population of "blue galaxies" seen only as fuzzy blobs in ground based images (Butcher \& Oemler 1978, 1984). Hubble Space Telescope (HST) images reveal that these "fuzzy blue blobs" are low luminosity, often disturbed, spiral galaxies "Sp" (Dressler et al 1994a,b, Couch et al 1994). Today, rich galaxy clusters are dominated by elliptical "E" and lenticular "S0" galaxies (Dressler 1980), mostly low luminosity dwarfs.

A successful model for the "Butcher-Oemler effect" has three requirements: 1) a mechanism for creating disturbed galaxies with enhanced star formation, 2) a cosmological context that explains why the mechanism operates most efficiently at $z \sim 0.4$, and 3 ) an identification of the remnants of the distorted blue galaxies in clusters today. Kauffman (1995) showed that hierarchical clustering models produce an enhancement at $z \sim 0.4$ given a mechanism that operates when a spiral first enters a cluster, handling the second requirement. Several mechanisms have been examined qualitatively: mergers (Icke 1985, Miller 1988), compression of gas in the high pressure cluster environment (Dressler \& Gunn 1983, Evrard 1991) and tidal compression by the cluster (Byrd and Valtonen 1990, Valluri 1993). Each of these mechanisms can produce starbursts, but none address morphological evolution and identify remnants. By analyzing their HST images, Oemler et al (1995) conclude that merging is implausible as the blue galaxy fraction is large and the merging probability is low. They observed disturbed spirals throughout the cluster, whereas both ram pressure stripping and global tides will only operate efficiently near the cluster's center. 


\section{GALAXY HARASSMENT AND CLUSTER EVOLUTION}

Although direct mergers are extremely rare, every galaxy experiences a high speed close encounter with a bright galaxy once per Gyr. The masses of bright galaxies in clusters will determine the havoc wreaked by these encounters. Galaxies in the field have massive dark halos, but there was speculation that these were stripped from individual galaxies within clusters (c.f. White and Rees 1978). Recently, Moore, Katz and Lake (1995) examined all of the physical processes that strip mass from galaxies within clusters. All galaxies are tidally limited by the potential field of the cluster. Over the 5 Gyr life of a cluster of galaxies, they find that bright galaxies retain most of the mass within their tidal radius (defined at the pericenter of their orbit), the rest being liberated by fast encounters with other bright galaxies. Hence, an $L_{*}$ galaxy in a rich cluster with a pericenter of 300 kpc will have a total mass of $\sim 4 \times 10^{11} M_{\odot}$. A rapid encounter with such a galaxy causes a tidal compression of the stellar and dark components. While these encounters are the cause of the stripping of mass from the dark halo (considered self-consistently throughout this work), their effect on small galactic disks is even more dramatic. The differential impulse violently redistributes the orderly motions of gas into non-circular intersecting orbits that promote star formation.

As clusters form, the gas rich disks of newly infalling spirals experience fast fly-by collisions when they enter the dense cluster environment. They are strongly perturbed resulting in disturbed morphologies and rapid bursts of star formation. To distinguish this from other collisional effects such as galaxy mergers and galaxy cannibalism, we refer to this process as "galaxy harassment". We use numerical simulations to follow the evolution of a small bulgeless spiral galaxy orbiting a dense cluster modeled on Coma. Our simulations use smoothed particle hydrodynamics (TREESPH, c.f. Hernquist \& Katz 1989) to evolve the gas component of the disk at resolutions of $100-500$ pc. Further details are found in MKL95.

We simulated galaxies on circular and elliptical orbits in smooth cluster potentials before examining the effects of harassment. The disk galaxy shows little evolution over 5 Gyrs when placed on a $450 \mathrm{kpc}$ circular orbit in a smooth cluster potential. The disk becomes bar unstable after the first pericentric passage (c.f. Byrd and Valtonen 1990). Thereafter, each time the galaxy passes through pericenter, the halo loses a small fraction of its mass but stars and gas remain bound. The most dramatic evolution occurs when we include the fast fly-by collisions of other galaxies. In these simulations, the galaxy has strong encounters throughout its evolution. The first encounter leads to a pronounced bar instability, but continued heating of the disk by perturbers results in a variety of new effects: morphological 
transformation, strong flow of gas into the center of the galaxy and creation of stellar/gaseous debris arcs.

One observational puzzle has been the ubiquity of disturbed galaxies with no sign of current interaction (Dressler et al 1994a). This feature is clearly seen in our simulated images. Over the course of $3 \mathrm{Gyr}$, the closest approach of any encounter is more than $30 \mathrm{kpc}$ away. Since the relative velocity of strong encounters is $\sim 1,500 \mathrm{~km} \mathrm{~s}^{-1}$, and the velocity impulse internal to the galaxy is only $\lesssim 50 \mathrm{~km} \mathrm{~s}^{-1}$, the perturbing galaxy moves $\sim 100 \mathrm{kpc}$ by the time that disk's response is visible. The bulk of the evolution is driven by $\lesssim$ five strong encounters with galaxies brighter than $\sim L_{*}$. As a result, the evolution is chaotic: whereas one fragile disk galaxy can avoid strong encounters for a few Gyr, another may be completely destroyed.

After several strong enounters, the loss of angular momentum to their own dark halos and the perturbing galaxies, combined with impulsive heating, leads to a prolate figure supported equally by random motions and rotation. The gas sinks to the very center of the galaxy and the stellar distribution is heated to the extent that it closely resembles a dwarf elliptical, although some retain very thick stellar disks and would be classed as dwarf lenticulars. Encounters cease to create sharp distortions and fail to remove any more material from the compact remnant.

Below $L_{*}$, two distinct classes of elliptical galaxies are observed. Low luminosity Es with high central surface brightness are a rare extension to the sequence of bright ellipticals; the archetype is M32. The most numerous galaxies in clusters are in a second class of dwarf ellipticals, also known as dwarf spheroidals (dE/dSph) - at least 3 magnitudes below $L_{*}$. Their exponential surface brightness profiles resemble those of spirals, as does the correlation of their low central surface brightnesses with total luminosity (Kormendy 1985).

The final stellar systems have a large degree of rotational support, surface density profiles and shapes that are in good agreement with observations (Ferguson \& Binggeli 1994 and references therein). The observed stellar populations of $\mathrm{dE}$ galaxies implies recent star formation activity that can easily be understood in our model as a result of recent encounters with cluster galaxies. Harassed Sd spiral galaxies undergo a remarkable transformation between morphological classes without any merging taking place. Their dynamical states can account for all of the dissimilarities between dwarf elliptical and normal elliptical galaxies. Harassment provides the link between the dominant populations of galaxies in clusters at $z \sim 0.4$ and the present-day. 


\section{Discussion}

G. Illingworth It would be very interesting to correlate the angular momentum and energy changes in the test galaxy with the perturber relative velocity to see if the slower encounters were those causing the biggest changes.

If the encounters are all in the impulsive regime then the global energy change is proportional to $M_{\text {pert }}^{2} / v_{\text {pert }}^{2} / b_{\text {impact }}^{4}$, although the response in the disk will depend on other factors.

B.M. Poggianti Could you outline the main similarities and differences between your "harassment" simulations and those that Barnes showed before? In particular, could you distinguish observationally between harassment and merging?

Barnes's merger simulations are between bound galactic systems, whereas rapid "fly-by" encounters drive the morphological evolution in clusters, where galaxies rarely come within $30 \mathrm{kpc}$ of each other. Preliminary work indicates that the harassed remnants are prolate and have a large degree of rotational support, unlike merger remnants which are typically oblate and pressure supported.

Acknowledgments This research was funded by NASA through the LTSA and HPCC/ESS programs.

\section{References}

Byrd G. \& Valtonen M. 1990, Ap.J., 350, 80-94.

Butcher H. \& Oemler A. 1978, Ap.J., 219, 18-33.

Butcher H. \& Oemler A. 1984, Ap.J., 285, 426-38.

Couch W.J., Ellis R.S., Sharples R. \& Smail I. 1994, Ap.J., 430, 121-38.

Dressler A. 1980, Ap.J., 236, 351-65.

Dressler A. \& Gunn J.E. 1983, Ap.J., 270, 7-19.

Dressler A, Oemler A., Butcher H. \& Gunn J.E. 1994a, Ap.J., 430, 107-20.

Dressler A, Oemler A., Sparks W.B. \& Lucas R.A. 1994b, Ap.J.Lett., 435, L23-

Evrard A.E. 1991, M.N.R.A.S., 248, 8p-10.

Ferguson H.C. \& Binggeli B. 1994, Astronomy and Astrophysics Review, 6, 67-1

Hernquist L. \& Katz N. 1989, Ap.J.Supp., 70, 419-46.

Icke V. 1985, A.A., 144, 115-23.

Kauffmann G. 1995, M.N.R.A.S., 274, 153-60.

Kormendy J. 1985, Ap.J., 295, 73.

Miller R.H. 1988, Comment. Astrophys., 13, 1-11.

Moore B., Katz N. \& Lake G. 1995, Ap.J., in press.

Oemler A. 1995, This proceedings.

Valluri M. 1993, Ap.J., 408, 57-70.

White S.D.M. \& Rees M. 1978, M.N.R.A.S., 183, 341. 